\title{
Transition from drop deposition to drop rebound during drop chain impact onto a hot target
}

\author{
J. Benedikt Schmidt*, Jan Breitenbach, Ilia V. Roisman, Cameron Tropea, Jeanette Hussong \\ Institute for Fluid Mechanics and Aerodynamics, Technische Universitaet Darmstadt, \\ Alarich-Weiss-Straße 10, Darmstadt, 64287, Germany \\ *Corresponding author email: schmidt@sla.tu-darmstadt.de
}

\begin{abstract}
Spray/wall interactions and single drop impact phenomena have been extensively studied in the past to better understand the underlying hydrodynamics and thermodynamics due to their high number of applications. One of these applications is a cooling by a chain of drops. The influence of many factors, such as impact parameters, material properties, surface temperature and liquid properties have already been largely identified. Various drop impact regimes, including nucleate boiling and film boiling, have been observed and described.

In this study the cooling of a hot substrate by an impacting monodispersed chain of drops is examined. The temperature distribution inside the substrate and its evolution in time are measured with a set of thermocouples. Additionally, a theoretical model is developed for the transient cooling of a semi-infinite solid target, allowing prediction of the increment of the wall temperature due to cooling at a single point. The theoretical prediction agrees well with the experimental data.
\end{abstract}

\section{Keywords}

drop chain, spray cooling, drop impact, heat transfer, transition boiling.

\section{Introduction}

It is now well established that spray cooling offers one of the most effective methods of cooling at high temperatures and for achieving high heat flux densities. Thus, spray cooling is already a wide-spread technology in applications such as metal production [1-3], cooling of electronic modules [4,5] or cryogenic cooling of human tissues [6]. Despite this extensive application of spray cooling technology, the system design and choice of operational parameters remain in most instances a highly empirical exercise, primarily due to the lack of an analytic basis on which to compute heat transfer rates as a function of all influencing parameters. Nevertheless, significant advances have been made in recent years, as outlined in a number of recent review articles [7, 8]. Hereby, it is important to distinguish between steady state cooling, in which the cooled substrate remains approximately at the same temperature, and transient cooling, in which the substrate temperature decreases in time [9]. Especially in the latter case, both the hydrodynamics and thermodynamics involved in the impact of individual drops vary tremendously, depending on which regime is currently prevailing. The possible regimes are generally associated with either the substrate superheat or the heat transfer coefficient and range from deposition and evaporation, nucleate boiling, transition boiling, thermal atomization [10] and film boiling. Thus, to properly predict heat flux densities in either steady state or transient boiling, the physics involved in each regime must be fully understood.

The drop impact leads to a temperature drop at the surface and a thermal gradient inside the substrate. The resulting heat flux inside the substrate can be described by the heat conduction equation inside the wall.

In the nucleate boiling regime the contact temperature at the liquid-solid interface can be assumed to be the saturation temperature of the liquid. The heat flux removed at the solid-liquid 
interface is therefore

$$
\dot{q}=\frac{e_{\mathrm{w}} \Delta T_{\mathrm{w}}}{\sqrt{\pi t}} .
$$

as described by [11], where $e_{\mathrm{w}}$ is the thermal effusivity of the solid substrate, with the wall temperature $T_{\mathrm{w}}$, time $t$, thermal diffusivity of the wall $\alpha_{\mathrm{w}}$. Balancing the total heat required to evaporate the drop and the heat transferred from the substrate, the residence time in the drop deposition regime can be described as

$$
t_{\text {deposit }}=\pi\left(\frac{\rho L^{*} D_{0}}{12 k_{\mathrm{w}} e_{\mathrm{w}} \Delta T_{\mathrm{w}}}\right)^{2}, \quad \Delta T_{\mathrm{w}}=T_{0}-T_{\mathrm{sat}},
$$

with the liquid density $\rho$, the sensible and latent heat $L^{*}$, saturation temperature of the liquid $T_{\text {sat }}$ and coefficient $k_{\mathrm{w}} \approx 1.6$ which is determined primarily by the surface structure and wettability [11].

At higher temperatures the residence time decreases more rapidly. An increasing amount of liquid rebounds, similar to the delayed drop rebound regime. Since less liquid is evaporated during the first impact of the drop at the substrate, the residence time decreases faster then described by Eq. (2).

From a certain temperature on, the bubbles create larger vapour clusters, leading to drop rebounds, even though the drops initially still wet the substrate. The residence time of drops at the surface is of the order of the natural oscillation time of drops

$$
t_{\sigma} \approx \sqrt{\frac{\rho D_{0}^{3}}{\sigma}}
$$

with $\sigma$ being the liquid/air surface tension.

The cooling of a small areas at the surface of a solid substrate, for example for an intensive cooling of small but powerful electronic devices, is typically performed using the chain of drops [12], impact of liquid micro jets [13] or array of drops [14]. The heat conduction in such systems leads to the temporal evolution of a three-dimensional temperature field, as soon as the thickness of the thermal boundary layer is much smaller than the size of the target. This problem differs therefore from the spray cooling problem, which is approximately one dimensional,

In this study the impact of a chain of drops onto a heated substrate is considered. The heat transfer is characterized experimentally. The experiments are also used for the validation of the theoretical model.

\section{Experimental Setup}

The experimental setup is shown schematically in Fig. 1. The setup comprises a drop generator, a heated surface with the thermal control system and a high-speed visualization system. The

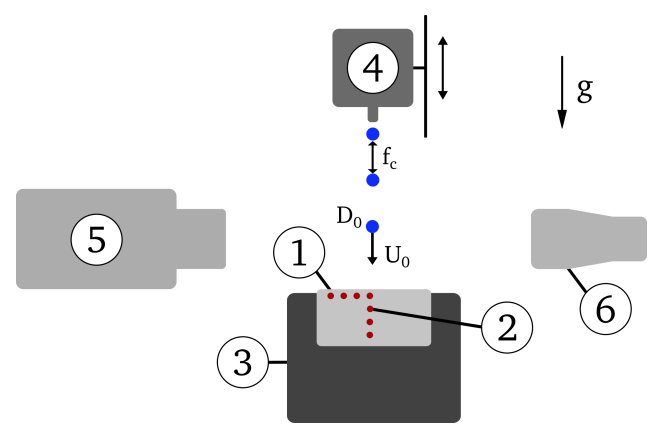

Figure 1. Sketch of the experimental setup. The impact target (1) is equipped with four radially and seven horizontally placed thermocouples (2). The impact target is placed inside a custom heater with an adiabatic insulation (3). The drops of the drop chain are generated with a syringe pump (4) and a constant volume flow rate to the needle. The impacts are observed from the side with a high-speed camera and back light shadowgraphy (5). 


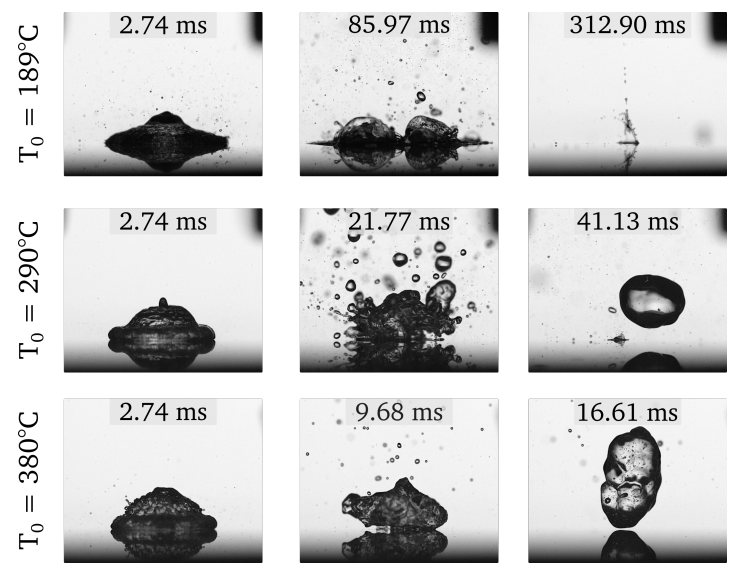

Figure 2. Typical drop impact regimes: drop deposition at the surface temperature $T_{0}=189{ }^{\circ} \mathrm{C}$; delayed drop rebound regime at $T_{0}=290^{\circ} \mathrm{C}$; and the drop rebound at $T_{0}=380^{\circ} \mathrm{C}$.

drop generator is used to generate single drops and/or a constant chain of drops. The liquid used in the experiments is double distilled water. Drops with a diameter of $D_{0}=2.2 \mathrm{~mm}$ are generated by dripping from a hydrophobic blunt needle. The liquid temperature is kept constant at $20^{\circ} \mathrm{C}$ using a temperature bath and a needle cooler. By applying a constant volume flow rate to the needle using a syringe pump, a constant chain of drops is achieved. The drop frequency $f_{c}$ is varied in the range of $0.9 \mathrm{~Hz}$ up to $5.7 \mathrm{~Hz}$. The impact velocity $U_{0}$ is controlled in the range of $U_{0}=0.45 \mathrm{~m} \mathrm{~s}^{-1}$ up to $1.25 \mathrm{~m} \mathrm{~s}^{-1}$ by adjusting the height of the needle above the impact surface. The impact surface is a $25 \mathrm{~mm}$ thick cylindrical stainless steel (1.4841) target with a diameter of $50 \mathrm{~mm}$. The impact surface of the target is mirror polished with a surface roughness of $S_{a}=10.8 \mathrm{~nm}$. The target is placed on a custom heater to heat up the impact surface up to $T_{0}=550^{\circ} \mathrm{C}$. The impacting drops cause a three-dimensional temperature gradient inside the target. Eleven type $J$ thermocouples (class 1 ) with a diameter of $0.5 \mathrm{~mm}$ are placed inside the target to measure the temperature gradient radially and normal to the surface. Based on the temperature gradient normal to the surface, the heat flux is calculated using a one-dimensional analytic solver provided by Woodfield et al. [15].

The substrate is heated to $T_{0}=550{ }^{\circ} \mathrm{C}$ for the drop chain experiments. After the system is in steady state, the heating system is switched off and a constant drop chain is applied to cool down the impact target. The drop chain is applied until the target is cooled down to a surface temperature of $T_{0} \approx 140{ }^{\circ} \mathrm{C}$. The experiments are repeated three times for each parameter setting. Even though the repetitions exhibit good agreement with one another, the heat flux is averaged as a function of the surface temperature.

To neglect the influence of natural convection as well as thermal radiation, the experiments have been repeated without any drop chain. The resulting measured heat flux is subtracted from the experimental results obtained with a drop chain, as a function of the surface temperature.

The drop impacts are recorded from the side with a high-speed camera at a frame rate of $6200 \mathrm{fps}$. A telecentric lens in combination with a telecentric LED are used for backlight shadowgraphy. The optical resolution of the system is $20 \mu \mathrm{m} /$ Pixel in the object plane. The system is used to measure the drop diameter $D_{0}$, impact velocity $U_{0}$, drop chain frequency $f_{c}$ and residence time $t_{r}$ of the drops on the surface. The residence time is understood as the time within no gap is visible between the drop liquid and the surface.

\section{Single drop observations}

Different drop impact regimes can be observed, depending on the initial surface temperature $T_{0}$. Side-view observations and the residence time of single drops impacting within different drop impact regimes are shown in Fig. 2 for an impact velocity of $0.46 \mathrm{~m} \mathrm{~s}^{-1}$.

For low surface temperatures, single drops deposit at the surface and evaporate. Depending on 

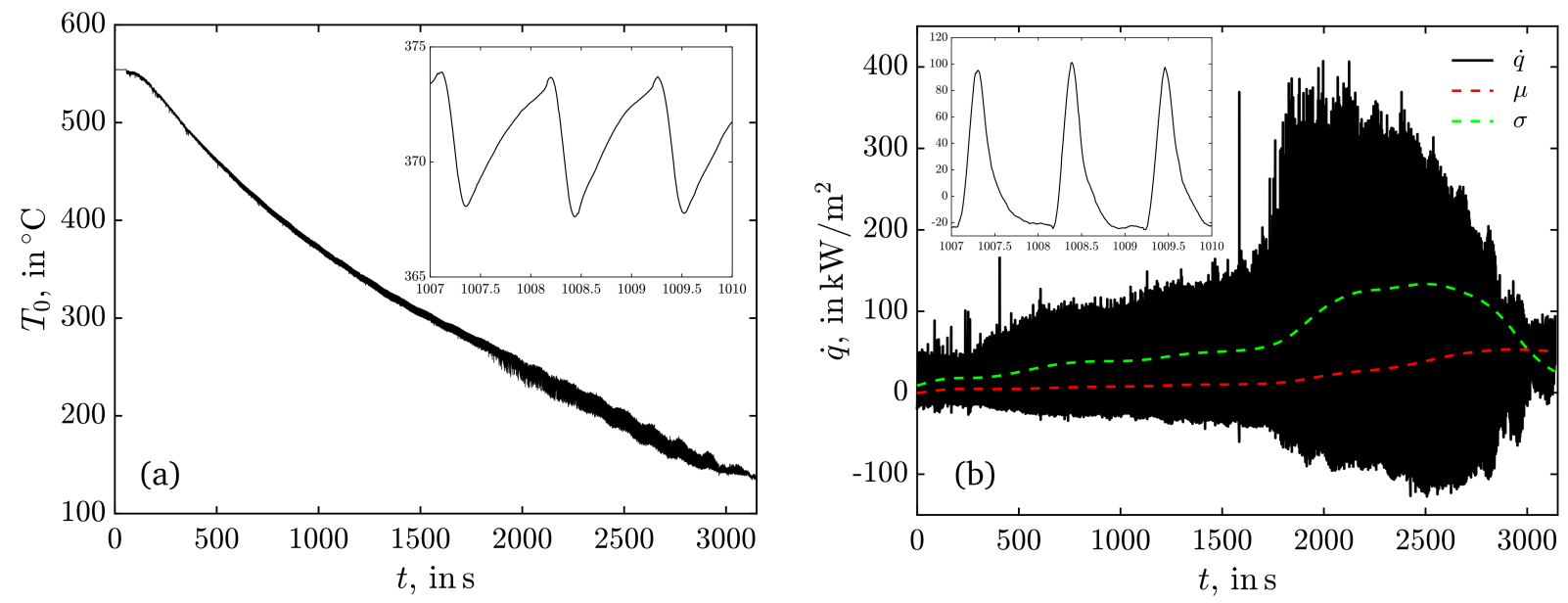

Figure 3. Diagram (a) shows the temperature decrease during the impact of a drop chain with a frequency $f_{c}=1 \mathrm{~Hz}$ onto a stainless steel target with $0.8 \mathrm{~m} \mathrm{~s}^{-1}$. The temperature oscillates due to the temperature decrease of each subsequent impacting drop, as shown in the detailed view. Diagram (b) shows the heat flux removed from the substrate. The black line shows the variation of the heat flux caused by each single drop impact, as shown in the detailed view. The dashed red line shows the moving average heat flux. The dashed green line indicates one standard deviation of the heat flux, given to qualitative evaluate the variations.

the current surface temperature, the drop collision can be accompanied by nucleate boiling, a delayed drop rebound [16] or can lead to the complete drop rebound.

\section{Drop chain: characterization of the thermal effects}

A drop chain impacting onto a hot substrate leads to a continuous cooling down of the substrate. The surface temperature during a drop chain impact is exemplary shown in Fig. 3(a). Beginning at $550{ }^{\circ} \mathrm{C}$ the heater is switched off and a drop chain is applied to the substrate. The drops impact with a frequency of $1 \mathrm{~Hz}$ and an impact velocity of $0.8 \mathrm{~m} \mathrm{~s}^{-1}$ at the substrate. The surface temperature decreases over time due to drop impacts, as well as to natural convection in the time between drop impacts. The heat loss due to natural convection becomes less with decreasing surface temperature. Nevertheless, the surface temperature continuously decreases and temperature oscillations increase in amplitude. At the end of the experiments, at low surface temperatures, the oscillations diminish.

These temperature oscillations are caused by the impacting drops. During the drop impact the surface temperature is lowered to the vapour-solid or liquid-solid contact temperature and a thermal gradient is induced inside the substrate as described by Eq. (1) for the nucleate boiling regime. In Fig. 3(a) a section of the inverse calculated surface temperature during drop impacts in the drop rebound regime is shown. Each drop impact causes a strong temperature drop. After the drop rebounds the surface slowly reheats from the hot substrate.

The heat flux removed from the substrate can be calculated based on the temperature decrease of the substrate, as described in the experimental methods. The temperature decrease arising from each single drop causes a high heat flux removal from the substrate.

The heat flux $\dot{q}$ is shown in Fig. 3(b). Each single drop causes a peak in the heat flux, as shown in the detailed view, followed by a slow reheating of the surface. The oscillations of the heat flux is shown in the plot as a black shaded area. The red dashed line represents the moving average of the heat flux $\mu$, which is equivalent to the heat flux of the drop chain. The dashed green line indicates one standard deviation $\sigma$, representing how strong the heat flux and surface temperature vary.

A vapor layer or vapour clusters insulate the drop from the substrate in the drop rebound regime at the high surface temperatures existing at the beginning of the experiment. The resulting heat flux oscillates in the range of $-20 \mathrm{~kW} \mathrm{~m}^{-2}$ to $100 \mathrm{~kW} \mathrm{~m}^{-2}$, since each drop impacts onto a dry hot substrate. The averaged heat flux is low in this regime. 
After approximately $1700 \mathrm{~s}$ the heat flux amplitude variation increases, indicating a change in the drop impact dynamics and contact time. The drops now wet the substrate, leading to a lower contact temperature, a higher temperature gradient inside the substrate and thereby a higher variation of the heat flux. Since the residence time of the drops is still short, the substrate can reheat between the residence time of the drops. Consequently the standard deviation of the heat flux increases significantly. Since the residence time is still short, the average heat flux increases only slowly but continuously.

After 2400 s the variation of the heat flux and standard deviation decreases, while the average heat flux still rises. The longer residence time leads to less time for the surface to reheat.

For low surface temperatures the drops start to impact onto small, non-boiling liquid residuals of previous drops at the substrate. While the residence time further increases, the mutual interaction of drops is enhanced. The surface temperature and heat flux fluctuates less, while the average heat flux continues to rise. The fluctuations allow to identify from which time on, or at which surface temperature the impacting drops start to interact.

Although a temperature decrease following each drop impact can be measured for low drop chain frequencies, the temperature values should be understood qualitatively and be averaged to analyse an overall temperature drop due to the impact of the drop chain. Faster measurement techniques, like high-speed IR cameras, should be used for single drop impacts and for more precise analysis of the contact temperature.

\section{A model for drop-chain cooling}

Consider the impact of a single liquid drop onto a solid substrate. A times much larger than the residence time of the drop, the impact can be approximated as an instantaneous event occurring in a single point at the wall surface. The perturbation of the temperature field in the wall caused by the drop impact has to satisfy the total energy balance $Q=2 \pi \rho c_{P} \int_{0}^{\infty} r^{2} T(r, t) \mathrm{d} r$, where $Q$ is the constant total heat transferred during the short collision event. The temperature field $T(r, t)$ also has to satisfy the zero heat flux condition at the wall interface at the times $t>0$ larger than the drop residence time.

The analytical solution of the heat conduction equation $\partial \Delta T / \partial t-\alpha \nabla^{2}(\Delta T)=0$ is assumed in the form $\Delta T=A t^{w} f(r / \sqrt{\alpha t})$, where $A$ and $w$ are unknown constants, $r$ is the distance from the origin and $r / \sqrt{\alpha t}$ is a similarity variable. The solution which satisfies the energy balance and the boundary conditions can be obtained only if $w=-3 / 2$. The corresponding solution of the heat conduction equation then takes the form

$$
\Delta T(r, t)=\frac{Q}{4 \pi^{3 / 2} \alpha^{3 / 2} \rho c_{P} t^{3 / 2}} \exp \left[-\frac{r^{2}}{4 \alpha t}\right] .
$$

Consider the impact of a chain of drops with the time period of drop impacts denoted $\Delta \tau$. The number of impacting drops before instant $t$ is $N=\operatorname{floor}(t / \Delta \tau)+1$, noting that the first drop impacts at $t=0$.

The corresponding temperature at time $t$ is obtained by the superposition of the temperature perturbations by $N$ drops, following Duhamel's theorem [17]

$$
\Delta T(r, t)=\frac{1}{4 \pi^{3 / 2} \alpha^{3 / 2} \rho c_{P}} \sum_{j=0}^{N-1} \frac{Q_{j}}{\left(t-\tau_{j}\right)^{3 / 2}} \exp \left[-\frac{r^{2}}{4 \alpha\left(t-\tau_{j}\right)}\right], \quad \tau_{j}=j \Delta \tau .
$$

Here $\tau_{j}$ is the instant of impact of the $j$-th drop and $\Delta T$ is the temperature increment due to drop impacts in relation to the initial wall temperature. In the case of significant convection, $\Delta T$ is the temperature increment in relation to the the temperature evolution due to the heat convection without drop impact. 
This expression can be approximated by an integral expression in the case for $N \gg 1$

$$
\Delta T(r, t) \approx \frac{1}{4 \pi^{3 / 2} \alpha^{3 / 2} \rho c_{P} \Delta \tau} \int_{0}^{t-\Delta \tau} \frac{Q(\tau)}{(t-\tau)^{3 / 2}} \exp \left[-\frac{r^{2}}{4 \alpha(t-\tau)}\right] \mathrm{d} \tau .
$$

The estimated wall temperature $T_{i}(t) \equiv T(0, t)$ just before an impact of a drop is therefore

$$
\Delta T_{i}(t) \approx \frac{1}{4 \pi^{3 / 2} \alpha^{3 / 2} \rho c_{P} \Delta \tau} \int_{0}^{t-\Delta \tau} \frac{Q(\tau)}{(t-\tau)^{3 / 2}} \mathrm{~d} \tau .
$$

For a constant value of $Q(\tau)=Q$ we obtain for $t \gg \Delta \tau$

$$
\begin{aligned}
\Delta T & =\frac{Q}{2 r \pi \alpha \rho c_{P} \Delta \tau}\left(\operatorname{erf}\left[\frac{r}{2 \sqrt{\alpha \Delta \tau}}\right]-\operatorname{erf}\left[\frac{r}{2 \sqrt{\alpha t}}\right]\right) \\
\Delta T_{i} & =\frac{Q}{r \pi \alpha \rho c_{P} \Delta \tau}\left(\frac{1}{\sqrt{\Delta \tau}}-\frac{1}{\sqrt{t}}\right)
\end{aligned}
$$

The long-time approximation for the interface temperature of the wall, cooled by a drop chain is

$$
\Delta T_{i \infty}=\frac{Q}{r \pi \alpha \rho c_{P} \Delta \tau^{3 / 2}}
$$

In Fig. 4(a) an exemplary temperature evolution $\Delta T(t)$ at a single measurement point is shown as a function of time. The temperature is measured in the heated target at the distance $1 \mathrm{~mm}$ from the surface. The graph shows the first $500 \mathrm{~s}$ after the cooling is started. The experimental measured temperature $\Delta T_{\exp }(t)=T_{\text {chain }}(t)-T_{\text {conv }}(t)$ is shown relative to the convectional cooling as a black line. The predicted temperature $\Delta T_{\text {model }}(t)$ by Eq. (8) is shown by the dashed line. The experiments are performed in the film boiling regime. The heat flux transferred to drop during impact is determined by the drop diameter, impact velocity and the material properties of the wall and the liquid. In this study the value of $Q$ is estimated from the model for the film boiling of a single drop [8]. The dependence of $Q$ on the substrate temperature is relatively weak and in this study for simplicity assumed constant, estimated as an average predicted value at the considered in the experiments range of the temperatures.

As shown in Fig. 4(a) the agreement between the theory and the measurements of $\Delta T$ is very good only during the first $270 \mathrm{~s}$. At times $t>270 \mathrm{~s}$ the theory starts to significantly deviate from the experimental data. This deviation is explained by the fact that the theory is developed for a semi-infinite target. The typical size of the heated region can be estimated as $R \sim \sqrt{\alpha t}$. The model is valid only when $R$ is smaller than the target thickness $H$, namely for the times smaller than $H^{2} / \alpha$. In our experiments $H^{2} / \alpha \approx 200$ seconds, which is comparable with the time at which the theory deviates from the experimental data.

Similar comparisons of the predicted and measured $\Delta T$ are shown in Fig. 4(b) for various drop chain frequencies $f_{c}$ from $1 \mathrm{~Hz}$ to $5.7 \mathrm{~Hz}$ are represented by different colors in the figure. The experimental temperature increments $\Delta T$ (solid lines) again exhibit good agreement with the predicted temperatures (dashed lines) for each frequency $f_{c}$. The temperature increment increases with drop chain frequency, as predicted by the influence of $\Delta \tau$ in Eq. (8).

Some scatter in the data can be explained by the effect of the natural convection, which leads to the corresponding temperature drop. The measurements become less precise when the influence of the wall cooling by drop impacts and by the natural convection and other heat losses are comparable. The is the case in Fig. 4(b), corresponding to the smallest considered frequency for which the scatter of the data is comparable with the value of $|\Delta T|$.

Good agreement between the theory and the experiments indicates that the theoretical approach, proposed in this study, can be used for further generalizations which will allow prediction of the cooling of the targets by thin jets or drop chains when the heat flux depends on the substrate temperature. Moreover, the idea can be used also for the description of the substrates of finite thickness. 

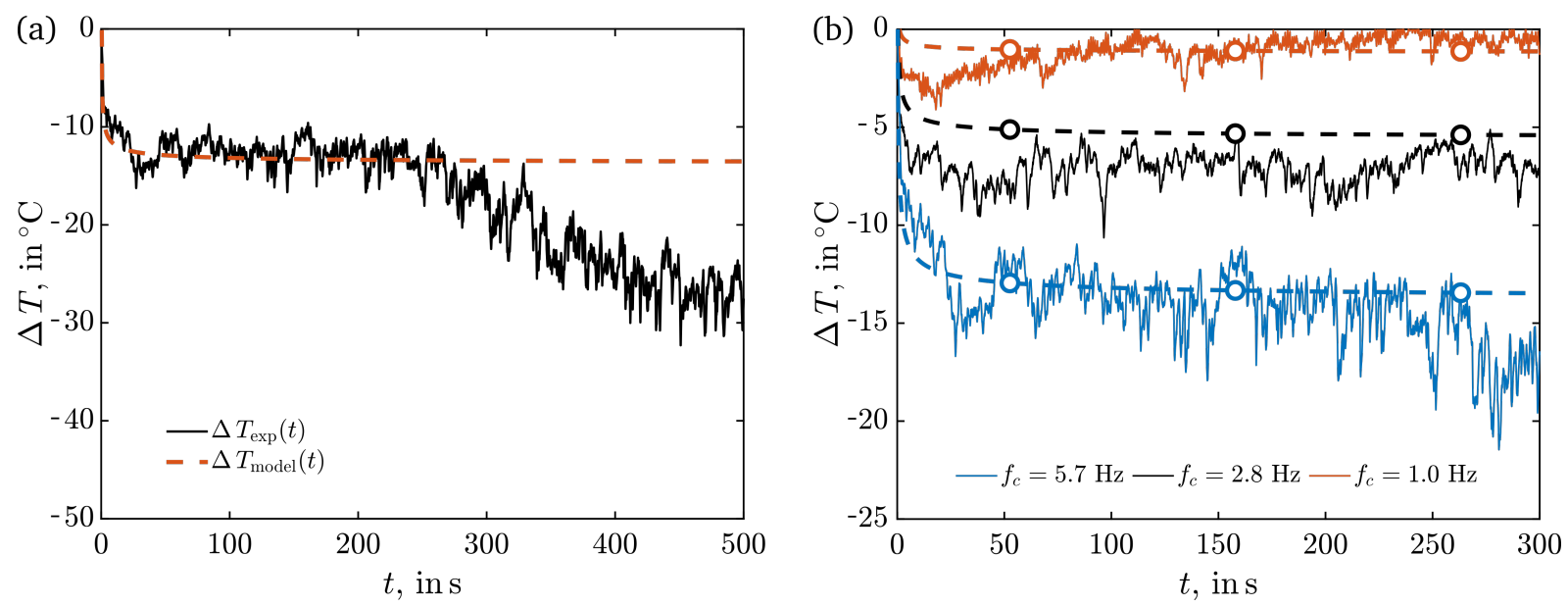

Figure 4. Comparison of the measured temperature increment $\Delta T(t)_{\exp }$ (solid line) with the theoretical predictions of Eq.8 $\Delta T(t)$ model (dashed line) inside the heated target $1 \mathrm{~mm}$ below the surface. In diagram (a) is an exemplary case with a drop chain frequency $f_{c}=5.7 \mathrm{~Hz}$ and the impact velocity $U_{0}=0.8 \mathrm{~m} \mathrm{~s}^{-1}$ shown for a time period of $500 \mathrm{~s}$. After $250 \mathrm{~s}$ the thermal boundary layer reaches the lower boundary of the heated target. For this reason the measured temperature increases faster then predicted by the model. In diagram (b) results for three different drop chain frequencies $f_{c}$ are shown. The colors represents the different $f_{c}$ from $1 \mathrm{~Hz}$ to $5.7 \mathrm{~Hz}$. The impact velocity $U_{0}$ is equal for all shown experiments. The experimental temperature increments correlate well with the theoretical predictions.

\section{Conclusions}

The present study investigated single drop as well as drop chain impacts onto hot surfaces with various impact velocities and drop chain frequencies. Depending on the surface temperature, different impact regimes with residence times have been observed during the single drop experiments.

An approximate theoretical model has been developed with the aim to predict the evolution of the wall temperature caused by an impact of a chain of drops. The model is based on an instantaneous source point of a finite amount of heat transferred from a substrate during drop impact. The model therefore is valid at the distances much larger than the drop diameter.

The theoretical predictions for the temperature evolution agree well with the experimental data. This model can be easily generalized to the cases of a continuous cooling by a single liquid micro-jet.

\section{Acknowledgements}

The authors gratefully acknowledge the Deutsche Forschungsgemeinschaft for its financial support in the framework of the Collaborative Research Center SFB-TRR 75, project number 84292822.

\section{Nomenclature}

$\begin{array}{lll}c_{\mathrm{P}} & \mathrm{J} \mathrm{K}^{-1} \mathrm{~kg}^{-1} & \text { specific heat capacity } \\ e_{\mathrm{w}} & \mathrm{J} \mathrm{K}^{-1} \mathrm{~m}^{-2} \mathrm{~s}^{-1 / 2} & \text { thermal effusivity } \\ D_{0} & \mathrm{~m} & \text { initial drop diameter } \\ f_{\mathrm{c}} & \mathrm{s}^{-1} & \text { drop chain frequency } \\ k_{\mathrm{w}} & - & \text { coefficient } \\ L^{*} & \mathrm{~J} \mathrm{~kg}^{-1} & \text { sensible and latent heat } \\ \dot{q} & \mathrm{~W} \mathrm{~m}^{-2} & \text { heat flux } \\ \dot{q}_{\mathrm{avg}} & \mathrm{W} \mathrm{m} \mathrm{m}^{-2} & \text { average heat flux } \\ Q & \mathrm{~J} & \text { heat removed by an impacting drop } \\ S_{\mathrm{a}} & \mathrm{m} & \text { average surface roughness } \\ t_{\mathrm{r}} & \mathrm{s} & \text { residence time } \\ t_{\mathrm{deposit}} & \mathrm{s} & \text { residence time during nucleate boiling }\end{array}$




$\begin{array}{lll}t_{\text {sigma }} & \mathrm{s} & \text { natural drop oscillation time } \\ T_{0} & { }^{\circ} \mathrm{C} & \text { surface temperature } \\ T_{1} & { }^{\circ} \mathrm{C} & \text { liquid temperature } \\ T_{\text {sat }} & { }^{\circ} \mathrm{C} & \text { saturation temperature } \\ \Delta T & { }^{\circ} \mathrm{C} & \text { temperature increment } \\ \Delta T_{\mathrm{w}} & { }^{\circ} \mathrm{C} & \text { wall super heat } \\ U_{0} & \mathrm{~m} \mathrm{~s}^{-1} & \text { drop impact velocity } \\ & & \\ \alpha & \mathrm{m}^{2} \mathrm{~s}^{-1} & \text { thermal diffusivity } \\ \rho & \mathrm{kg} \mathrm{m}^{-3} & \text { liquid density } \\ \sigma & \mathrm{N} \mathrm{m}^{-1} & \text { liquid surface tension } \\ \Delta \tau & \mathrm{s} & \text { time period of drop impacts }\end{array}$

\section{References}

[1] Chen, S.-J., Tseng, A. A., 1992, Int. J. Heat Fluid Flow, 13 (4) pp. 358-369.

[2] Mudawar, I., Deiters, T. A., 1994, Int. J. Heat Mass Transf., 37 (3) pp. 347-362.

[3] Aamir, M., Qiang, L., Hong, W., Xun, Z., Wang, J., Sajid, M., 2017, Appl. Therm. Eng., 115 pp. 418-434.

[4] Tilton, D. E., Kearns, D. A., Tilton, C. L., 1994, in Kittel, P., ed., Advances in Cryogenic Engineering, Springer US, Boston, MA, pp. 1779-1786.

[5] Bar-Cohen, A., Arik, M., Ohadi, M., 2006, Proceedings of the IEEE, 94 (8) pp. 1549-1570.

[6] Datrice, N., Ramirez-San-Juan, J., Zhang, R., Meshkinpour, A., Aguilar, G., Nelson, J. S., Kelly, K. M., 2006, Dermatol. Surg., 32 (8) pp. 1007-1012.

[7] Mudawar, I., 2013, J. Therm. Sci. Eng. Appl., 5 (2) p. 021012.

[8] Breitenbach, J., Roisman, I. V., Tropea, C., 2018, Exp. Fluids, 59 (3) p. 55.

[9] Tenzer, F. M., Roisman, I. V., Tropea, C., 2019, J. Fluid Mech., 881 pp. 84-103.

[10] Roisman, I. V., Breitenbach, J., Tropea, C., 2018, J. Fluid Mech., 842 pp. 87-101.

[11] Breitenbach, J., Roisman, I. V., Tropea, C., 2017, Phys. Rev. Fluids, 2 (7) p. 074301.

[12] Lu, C.-F., Huang, C.-M., Yang, J.-C., Chiang, H.-L., Fu, C.-C., Cheng, C.-H., Chen, C.-J., 2005, MRS Online Proceedings Library Archive, 888.

[13] Wang, E. N., Zhang, L., Jiang, L., Koo, J.-M., Maveety, J. G., Sanchez, E. A., Goodson, K. E., Kenny, T. W., 2004, J. Microelectromech. Syst., 13 (5) pp. 833-842.

[14] Heffington, S., Black, W., Glezer, A., 2002, in ITherm 2002. Eighth Intersociety Conference on Thermal and Thermomechanical Phenomena in Electronic Systems (Cat. No. 02CH37258), IEEE, pp. 408-412.

[15] Woodfield, P. L., Monde, M., Mitsutake, Y., 2006, Int. J. Heat Mass Transf., 49 (17-18) pp. 2864-2876.

[16] Schmidt, J. B., Hofmann, J., Tenzer, F., Breitenbach, J., Tropea, C., Roisman, I., 2021, Nature Portfolio, Preprint (Version 1) available at Research Square [https://doi.org/10.21203/rs.3.rs-56255/v1], 2020, pp.553-562.

[17] Myers, G. E., 1987, Analytical methods in conduction heat transfer, Genium Publishing Corp., Schenectady, NY. 